\title{
Tracheobronchial foreign bodies in children - a retrospective study of 2,000 cases in Northwestern China
}

This article was published in the following Dove Press journal:

Therapeutics and Clinical Risk Management

28 August 2015

Number of times this article has been viewed

\author{
Jianmin Liang' \\ Juan $\mathrm{Hu}^{\prime}$ \\ Huimin Chang ${ }^{2}$ \\ Ying Gao' \\ Huanan Luo' \\ Zhenghui Wang' \\ Guoxi Zheng' \\ Fang Chen' \\ Ting Wang' \\ Yeye Yang' \\ Xiaohui Kou' \\ Min $X u^{\prime}$ \\ 'Department of Otolaryngology- \\ Head and Neck Surgery, The Second \\ Hospital, Xi'an Jiaotong University, \\ ${ }^{2}$ Department of Otolaryngology-Head \\ and Neck Surgery, Affiliated Hospital \\ of Xi'an Medical University, Xi'an, \\ People's Republic of China
}

\begin{abstract}
The aim of this study is to report our experience in the diagnosis and treatment of tracheobronchial foreign bodies (TFBs). We retrospectively reviewed medical records of 2,000 TFB patients (1,260 males and 740 females) who were treated between January 2010 and December 2013. Chest radiography and computed tomography were performed to diagnose TFBs. The location and type of foreign bodies (FBs), anesthesia methods, and treatment outcomes and complications were analyzed. Overall, $72.5 \%$ of our patients with TFB were aged between 1 years and 3 years. Plant-based FBs are the most common FB type, accounting for $91.5 \%$. Almost $52.1 \%$ of the FBs were encountered in the right bronchus. The coincidence rate for computed tomography-based three-dimensional reconstruction was significantly greater than that for chest $\mathrm{X}$-ray examination $(98.7 \%$ vs $82.0 \%, P<0.01)$. Under general anesthesia, the FBs were removed by rigid bronchoscopy. Neither anesthesia complication nor intraoperative hypoxemia occurred. There were seven deaths from acute obstructive asphyxia and eight from residual FB-induced chronic asphyxia and respiration-circulation failure. In conclusion, early diagnosis and prompt treatment of TFBs with rigid bronchoscopy under general anesthesia is effective in reducing complications and mortality in affected children.
\end{abstract}

Keywords: respiratory tract foreign body, children, diagnosis, treatment

\section{Introduction}

Tracheobronchial foreign body (TFB) is one of the most common pediatric emergencies. It occurs more commonly in children under the age of five, owing to their insufficient airway protection reflex, poor chewing ability, and harmful habits of exploring objects with mouth as well as eating while crying or playing. TFB may lead to serious complications such as airway inflammation, hemoptysis, bronchiectasis, pulmonary atelectasis, and even asphyxia and death. ${ }^{1-3}$ Nowadays, although the health care education has been propagandized, the incidence of TFB has not been reduced. TFB is the forth leading cause of accidental death for children aged less than 3 years. ${ }^{4}$ Early diagnosis and prompt treatment are the keys to reduce TFB-related complications and mortality. Chest radiography, computed tomography (CT), and rigid bronchoscopy are commonly used in the diagnosis of FB aspiration. ${ }^{5}$ Management of foreign body (FB) aspiration depends on the type and location of FB. Flexible or rigid bronchoscopy is the main method used to remove FBs in the airway. ${ }^{6,7}$ Tracheotomy is performed on rare occasions, for example, the FB is too large to pass the subglottic region, or is sharply pointed and risks perforating the airway. ${ }^{8}$ From January 2010 to December 2013, 2,000 children with TFB were treated in our hospital and their medical records were reviewed retrospectively. This study aims to report our experience in the diagnosis
Correspondence: Zhenghui Wang Department of Otolaryngology-Head and Neck Surgery, The Second Hospital, Xi'an Jiaotong University, No 157 Xi Wu Road, Xi'an, Shaanxi Province 7I0004, People's Republic of China

Tel +862987679866

Fax +862987678421

Email ehui4298@I63.com 
and treatment of TFBs and evaluated the location and type of FBs, anesthesia methods, outcomes, and complications.

\section{Materials and methods}

\section{Patients}

We retrospectively reviewed medical records of 2,000 TFB patients (1,260 males and 740 females) who were treated at the Second Affiliated Hospital of Xi' an Jiaotong University School of Medicine (Xi'an, People's Republic of China) between January 2010 and December 2013. Their median age was 2.4 years (range $0.3-12$ years). Inclusion criteria were as follows: 1) history of FB aspiration, 2) presence of TFB on the basis of imaging tests, and 3) recurrent cough or dyspnea. Patients with evidence of asthma were excluded from this study. All the 2,000 patients underwent chest X-ray examination and 1,500 of them underwent additional chest computed tomographic (CT) and three-dimensional reconstruction. Parental/guardian informed consent was obtained. This study was approved by the Institutional Review Board of Xi'an Jiaotong University School of Medicine.

\section{Treatment}

\section{Anesthesia technique}

All of the patients except three who were admitted under emergency conditions received surgical treatment after anesthesia with intravenous injection of ketamine and midazolam. The preoperative fasting time was 12 hours for solid food and 6 hours for beverage. Atropine $(0.01 \mathrm{mg} / \mathrm{kg}$ body weight), which was used to reduce bronchial secretion and prevent postoperative pneumonia, was intramuscularly injected 30 minutes before surgery. During anesthesia, sevoflurane was inhaled and $1 \%$ lidocaine was applied to the inner surface of the throat.

\section{Surgical technique}

Depending on clinical conditions, FBs were removed by various techniques, including rigid bronchoscopy, direct laryngoscopy, and tracheotomy followed by rigid bronchoscopy. The most common surgical technique applied to our patients was rigid bronchoscopy. Under general anesthesia, a rigid bronchoscope was inserted into the airway, and mechanical ventilation was reestablished by connecting an automated jet ventilation device (Aide Medical Instrument Company, Taicang, People's Republic of China) to the side arm of the rigid bronchoscope. Intraoperatively, the blood oxygen of patients was maintained $>80 \%$. After locating the site of FB obstruction, the bronchoscope was immediately positioned above the FB. A grasping forceps was introduced to remove the FB. After the successful retrieval of the FB, the rigid bronchoscope was reintroduced to confirm the complete removal of the foreign body.

\section{Statistical analysis}

Data analysis was performed using the SPSS Statistics 19.0 software (StataCorp LP, College Station, TX, USA). The $t$-test was used for comparison of measurement data that were presented by means \pm standard deviation. Enumeration data were expressed as percentage. $P<0.05$ was considered as statistical significance.

\section{Results \\ Clinical symptoms}

Overall, $72.5 \%$ of our patients with TFB were aged between 1 years and 3 years. Almost 1,105 of the 2,000 patients presented dyspnea, 653 cough, 215 hoarseness, three depression sign, cyanosis, and fever, and 27, asphyxia. Chest auscultation showed decreased or absent ipsilateral breath sounds (1,250 cases), rough breathing sounds (985 cases), rapid breathing (569 cases), and wheezing sounds (123 cases). Chest percussion revealed dullness (400 cases), dry or wet rales (56 cases), and no abnormal signs (18 cases).

\section{Results of radiological tests}

All our 2,000 cases underwent chest X-ray examination. In total, 1,350 of them showed signs of foreign bodies, 630 mediastinal swing, 290 obstructive pulmonary emphysema, 225 foreign bodies combined with objective pneumonia, whereas 15 cases showed negative signs. Of the 1,500 cases undergoing three-dimensional reconstruction from chest CT, 1,215 cases (81.0\%) showed direct signs of FB obstruction, which was confirmed by bronchoscopy, and $265(17.7 \%)$ showed indirect findings of FB reported as suspicious TFB. A representative case of TFB on chest CT (Figure 1) and X-ray (Figure 2) imaging is shown. Twenty cases $(1.3 \%)$ were misdiagnosed as bronchopneumonia (14 cases) or asthma (6 cases), which were corrected by bronchoscopy. The coincidence rate for CT-based threedimensional reconstruction was significantly greater than that for chest $\mathrm{X}$-ray examination $(98.7 \%$ vs $82.0 \%, P<0.01$; Table 1).

\section{FB type and location of obstruction}

The FBs were roughly classified into four types: plant-based FBs (91.5\%), bone-based FBs (1.2\%), plastic and metal FBs $(0.6 \%)$, and others $(6.7 \%)$. The FB was encountered in the right bronchus in 1,042 cases $(52.1 \%)$, the left bronchus in 


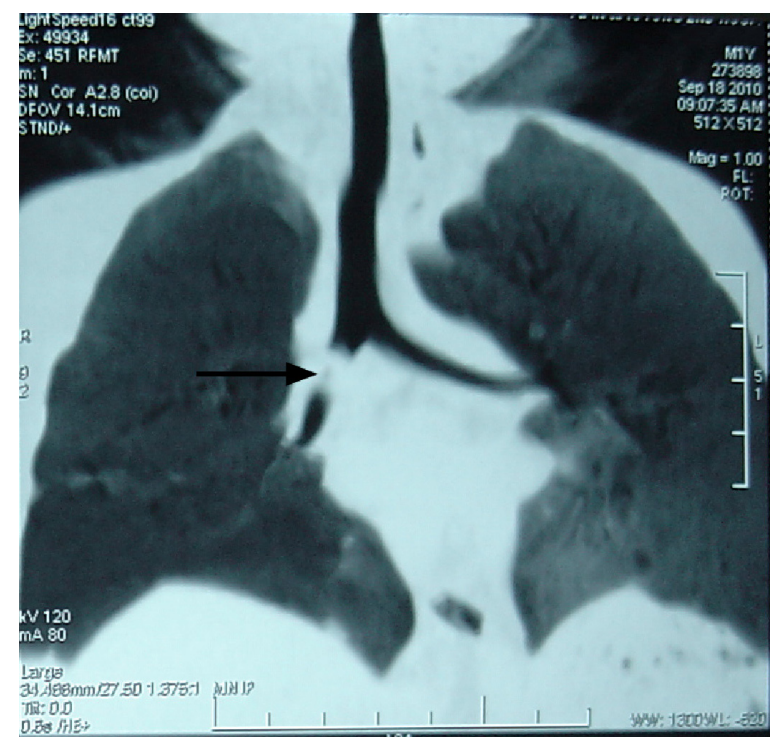

Figure I A representative case of TFB (arrow) detected by chest CT scan. Abbreviations: TFB, tracheobronchial foreign body; CT, computed tomography.

782 cases (39.1\%), subglottic larynx in 148 cases (7.4\%), and bilateral bronchus in 28 cases (1.4\%; Table 2$)$.

\section{Treatment outcomes and complications}

Of the 2,000 cases, 1,843 was removed by rigid bronchoscopy, 156 by laryngoscopy, and one case by thoracotomy (Table 2 ). Neither anesthesia complication nor intraoperative hypoxemia occurred. The patients underwent clinical review 1 week after surgery. Fifteen cases received second surgery to eliminate clinical symptoms of TFB. There were 15 deaths (all aged under 2 years). Causes of death included acute obstructive asphyxia (seven cases) and residual FB-induced chronic asphyxia and respiration-circulation failure (eight cases). A total of 421 cases were complicated by pneumonia and recovered 2-3 weeks after surgery. Forty cases were complicated by pulmonary atelectasis; one of them underwent lobectomy due to lung dysfunction and the other 39 cases recovered within 3 months. Additionally, there were 37 cases of laryngeal obstruction, 36 respiratory failures, 13 heart failures, 40 subcutaneous emphysemas, and 26 mediastinal emphysemas. These complications were resolved within 3 weeks after surgery.

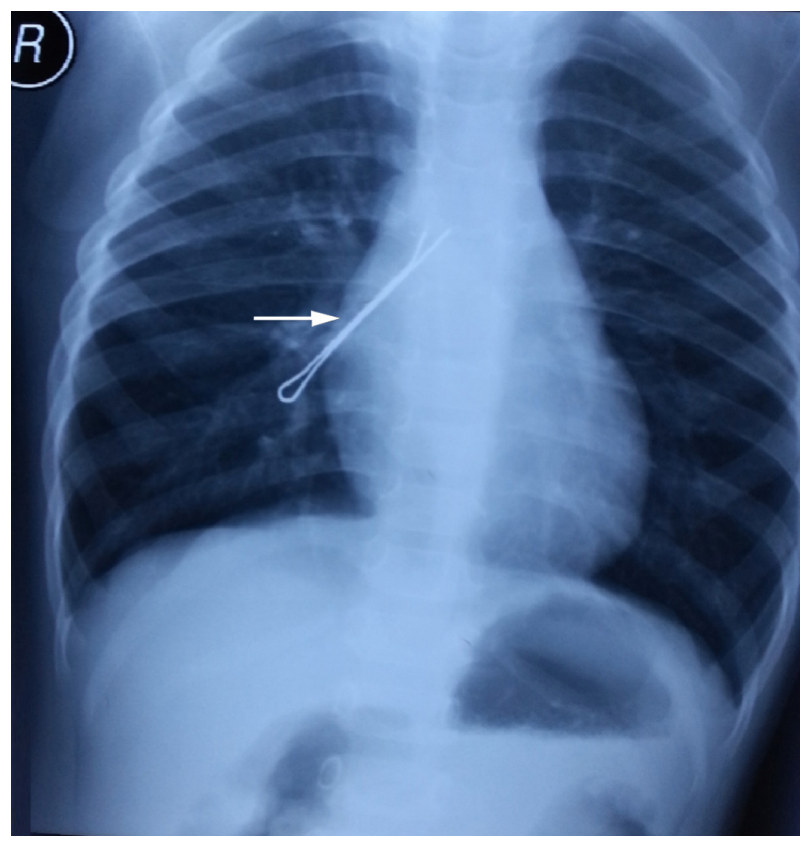

Figure 2 A representative case of TFB (arrow) detected by chest X-ray examination. Abbreviation: TFB, tracheobronchial foreign body.

\section{Discussion}

Due to lack of molar teeth, infants and young children have poor ability of chewing. Their airway protection reflex has not been well developed. Additionally, they are highly interested in and tend to explore environmental objects with mouth. ${ }^{9}$ These factors contribute to a high incidence of TFB in young children. In supporting this viewpoint, $72.5 \%$ of our patients with TFB were aged between 1 years and 3 years. Correct diagnosis is of paramount importance in treating inhaled FBs. Of our 2,000 patients, 1,880 had a clear history of FB aspiration, which contributed to early diagnosis of FBs. Chest $\mathrm{X}$-ray tests were applied to the remaining 120 patients who did not have a history of FB aspiration. However, a negative finding on chest X-rays cannot rule out TFB diagnosis. ${ }^{10}$ Some transparent FBs are not visible by X-ray imaging. Chest $\mathrm{X}$-ray test has a relatively low sensitivity in the identification of TFBs. It has been reported that chest CT scan shows a greater diagnostic sensitivity and specificity for TFBs than chest X-ray test. ${ }^{11}$ Consistently, our data revealed that

Table I Comparison of coincidence rate

\begin{tabular}{lllll}
\hline & $\mathbf{n}$ & Direct signs & Indirect findings & Coincidence rate (\%) \\
\hline 3-D reconstruction CT & $\mathrm{I}, 500$ & $\mathrm{I}, 215$ & 265 & 98.7 \\
Chest X-ray & 2,000 & $\mathrm{I}, 350$ & 290 & 82.0 \\
$\chi^{2}$ & - & - & - & 246.008 \\
$P$ & - & - & - & 0.000 \\
\hline
\end{tabular}

Abbreviations: 3-D, three-dimensional; CT, computed tomography. 
Table 2 Removal methods and location of FB

\begin{tabular}{|c|c|c|c|c|c|c|}
\hline & \multicolumn{3}{|c|}{ Removal methods } & \multicolumn{3}{|c|}{ Locations of obstruction } \\
\hline & Bronchoscope & Laryngoscope & Thoracotomy & Left bronchus & Right bronchus & Trachea \\
\hline Cases (n) & 1,843 & 156 & I & 782 & 1,042 & 148 \\
\hline Constituent ratio (\%) & 92.15 & 7.8 & 0.05 & 39.66 & 52.84 & 7.51 \\
\hline
\end{tabular}

Abbreviation: FB, foreign body.

three-dimensional reconstruction from chest CT was more powerful in diagnosing FBs than chest X-ray test. Therefore, we suggest that three-dimensional reconstruction from chest CT is useful in detecting TFBs, especially in cases without a history of FB aspiration. Among our patients, 20 cases were misdiagnosed as bronchopneumonia (14 cases) or asthma (six cases), which were corrected by bronchoscopy. Patients with TFB that is located at unilateral bronchioles causing atypical clinical symptoms are more likely to be misdiagnosed as airway infection or other diseases. The reasons for misdiagnosis mainly include 1) lack of a clear FB aspiration history, 2) negative findings on X-rays, and 3) poor experience on TFBs.

For cases with a definite diagnosis of TFB, prompt treatment usually via bronchoscopy is of importance. Anesthesia is a crucial factor affecting the procedure of bronchoscopy. ${ }^{12}$ Because anesthesia and surgery share the airway, it is hard to control the depth of anesthesia. Under emergency conditions, immediate bronchoscopy may be performed without anesthesia. However, general anesthesia makes patients unconscious, which is accepted to be more safe and convenient for surgery. In this study, all of the patients except three who were admitted under emergency conditions underwent general anesthesia before surgery, which should block autonomous breathing and thus avoid airway spasm. No anesthesia-related complications occurred.

For children with serious complications, such as fever, acidosis, and systemic failure, it is crucial to evaluate operation risk and patients' tolerance before surgery. During operation, the airway is ventilated continuously and bright lights are needed at all the time. A bronchoscope was advanced to the trachea when the glottis is open. ${ }^{13}$ Chest auscultation was performed frequently during surgery. Loss of breath sounds, sharp reduction of blood oxygen, and aggravated dyspnea are signs of pneumothorax, which needs to be immediately treated. For huge FBs, direct removal via bronchoscopy is not recommended; otherwise, it may lead to serious iatrogenic drama. In the current study, two cases of huge FBs were removed by tracheotomy. The first 24 hours after operation is a high-risk period that needs more attention, as long-term hypoxemia may cause cell edema, respiratory arrest, cardiac arrhythmia, or even sudden death.
Treatment of uncommon FBs is challenging. There were 16 patients with huge plastic FBs among the 2,000 patients studied. We developed several new methods and special instruments to deal with uncommon FBs. For instance, retrograde forceps are employed to remove hollow cylindershaped plastic FBs, and magnetic attraction is used to extract spherical metal FBs. To avoid blocking the glottis during the removal of FBs, we made incisions and exposed the pretracheal fascia without opening the trachea and afterward inserted the bronchoscope to remove FBs. Once the FB slipped or incarcerated, operators should make a tracheal incision immediately and remove the FB quickly.

\section{Conclusion}

TFBs present most frequently in children aged between 1 years and 3 years. Early diagnosis and prompt treatment of TFBs with rigid bronchoscopy under general anesthesia are of significance in reducing complications and mortality in affected children.

\section{Disclosure}

The authors report no conflicts of interest in this work.

\section{References}

1. Sahin A, Meteroglu F, Eren S, Celik Y. Inhalation of foreign bodies in children: experience of 22 years. J Trauma Acute Care Surg. 2013;74: 658-663.

2. Gregori D, Salerni L, Scarinzi C, et al; ESFBI Study Group. Foreign bodies in the upper airways causing complications and requiring hospitalization in children aged 0-14 years: results from the ESFBI study. Eur Arch Otorhinolaryngol. 2008;265:971-978.

3. Divisi D, Di Tommaso S, Garramone M, et al. Foreign bodies aspirated in children: role of bronchoscopy. Thorac Cardiovasc Surg. 2007;55: 249-252.

4. Rodríguez H, Passali GC, Gregori D, et al. Management of foreign bodies in the airway and oesophagus. Int J Pediatr Otorhinolaryngol. 2012; 76:S84-S91.

5. Tuckett P, Cervin A. Reducing the number of rigid bronchoscopies performed in suspected foreign body aspiration cases via the use of chest computed tomography: is it safe? A literature review. J Laryngol Otol. 2015;129(S1):S1-S7.

6. Saki N, Nikakhlagh S, Rahim F, Abshirini H. Foreign body aspirations in infancy: a 20-year experience. Int J Med Sci. 2009;6(6):322-328.

7. Mallick MS. Tracheobronchial foreign body aspiration in children: a continuing diagnostic challenge. Afr J Paediatr Surg. 2014;11(3): 225-228.

8. Tang CL, Lee SC, Mohamad Lal A, Thomas RA, Ngui LX, Lim LY. Tracheotomy: an alternative for tracheobronchial foreign body removal. Med J Malaysia. 2014;69(5):241-243. 
9. Higo R, Matsumoto Y, Ichimura K, Kaga K. Foreign bodies in the aerodigestive tract in pediatric patients. Auris Nasus Larynx. 2003;30: 397-401.

10. Baharloo F, Veyckemans F, Francis C, Biettlot M-P, Rodenstein DO. Tracheobronchial foreign bodies: presentation and management in children and adults. Chest $J$. 1999;115:1357-1362.

11. Sattar A, Ahmad I, Javed AM, Anjum S. Diagnostic accuracy of chest $\mathrm{x}$-ray in tracheobronchial foreign body aspiration in paediatric patients. J Ayub Med Coll Abbottabad. 2011;23(4):103-105.
12. Liu J, Xiao K, Lv X. Anesthesia and ventilation for removal of airway foreign bodies in 35 infants. Int J Clin Exp Med. 2014;7(12): $5852-5856$.

13. Oncel M, Sunam GS, Ceran S. Tracheobronchial aspiration of foreign bodies and rigid bronchoscopy in children. Pediatr Int. 2012;54: $532-535$.

\section{Publish your work in this journal}

Therapeutics and Clinical Risk Management is an international, peerreviewed journal of clinical therapeutics and risk management, focusing on concise rapid reporting of clinical studies in all therapeutic areas, outcomes, safety, and programs for the effective, safe, and sustained use of medicines. This journal is indexed on PubMed Central, CAS,
EMBase, Scopus and the Elsevier Bibliographic databases. The manuscript management system is completely online and includes a very quick and fair peer-review system, which is all easy to use. Visit http://www.dovepress.com/testimonials.php to read real quotes from published authors.

Submit your manuscript here: http://www.dovepress.com/therapeutics-and-clinical-risk-management-journal 